\title{
Fast fall-time ion beam in neutron generators
}

\author{
Q. Ji, J. Kwan, M. Regis, Y. Wu, S. B. Wilde, J. Wallig \\ Lawrence Berkeley National Laboratory, 1 Cyclotron Road, Berkeley, CA 94720, U. S. A
}

\begin{abstract}
Ion beam with a fast fall time is useful in building neutron generators for the application of detecting hidden, gamma-shielded SNM using differential die-away (DDA) technique. Typically a fall time of less than $1 \mu$ s can't be achieved by just turning off the power to the ion source due to the slow decay of plasma density (partly determined by the fall time of the RF power in the circuit). In this paper, we discuss the method of using an array of mini-apertures (instead of one large aperture beam) such that gating the beamlets can be done with low voltage and a small gap. This geometry minimizes the problem of voltage breakdown as well as reducing the time of flight to produce fast gating. We have designed and fabricated an array of 16 apertures $(4 \times 4)$ for a beam extraction experiment. Using a gating voltage of $1400 \mathrm{~V}$ and a gap distance of $1 \mathrm{~mm}$, the fall time of extracted ion beam pulses is less than $1 \mu$ s at various beam energies ranging between $400 \mathrm{eV}$ to $800 \mathrm{eV}$. Usually merging an array of beamlets suffers the loss of beam brightness, i.e., emittance growth, but that is not an important issue for neutron source applications.
\end{abstract}

Keywords: fast fall-time ion beam, neutron generator, differential die-away.

PACS: 41.75.Ak, 29.25.Dz

\section{INTRODUCTION}

Active neutron interrogation has been demonstrated to be an effective method of detecting shielded fissile material. The penetrating ability of neutrons allows them to "see" through material that may be surrounding the fissile material. When the neutrons interact with fissile material they induce fission resulting in the emission of neutrons and gammas that may then be detected. A fast falltime/fast pulsing axial neutron generator is needed primarily for differential die-away technique (DDA) interrogation systems. The DDA has been used for some time to measure the fissile content of nuclear waste containers, and is a sensitive technique for detecting the presence of fissile materials such as ${ }^{235} \mathrm{U}$ and ${ }^{239} \mathrm{Pu}$. In DDA analysis, a neutron generator produces repetitive pulses of neutrons that are directed into a cargo container that is under inspection. As each pulse passes through the cargo, the neutrons are thermalized and absorbed. The thermalization process is very rapid and the epithermal neutrons decays within microseconds. The thermal neutrons, however, decay much slower, which is on the order of hundreds of microseconds. If Special Nuclear Material (SNM) is present, the thermalized neutrons from the source will cause fissions that produce a new source of neutrons. These fast fission neutrons decay with a time very similar to that of the thermal neutron die-away of the surrounding cargo. A fast fall-off of the neutron pulse and low neutron background will improve the DDA signal and SNM detection. ${ }^{1,2}$

A compact neutron generator, with enhanced features such as sub-microsecond ion beam fall time and low background neutrons, is being developed in Lawrence Berkeley National Laboratory. The main performance characteristics are: (1) pulse width of tens of $\mu \mathrm{s}$; (2) pulse fall time of less than $1 \mu \mathrm{s}$; (3) repetition rate of $10 \mathrm{kHz}$; and (4) peak neutron yield $>10^{9} \mathrm{n} / \mathrm{s}$. The system will aim at both high and low beam current applications. In this paper, different methods of beam switching will be evaluated, in terms of fall-off time, reliability, and neutron background level. Experimental setup to obtain fall-time less than $1 \mu \mathrm{s}$ will be described and results will be discussed.

\section{COMPARISON OF DIFFERENT BEAM SWITCHING METHODS}

There are several schemes to achieve beam switching. We compare different methods in terms of the fall time of beam pulse, background noise, and system complexity.

\section{Beam Pulsing By Controlling RF Power}

The easiest way to obtain a pulsed beam is to simply switch on and off the plasma. ${ }^{3,4}$ An ion beam 
extracted from an ion source, such as a RF-driven plasma source, can be shut off by turning off the RF power supply. The fall time using this method is determined by the fall-time of the RF power supply coupled with a slow intrinsic plasma delay, which is also called the "after glow". Depending on the type of RF power supply, the typical fall time is around tens of microseconds.

\section{Beam Pulsing By Switching The High Voltage}

Another way to get a pulsed beam is to pulse the acceleration high voltage while keeping the plasma running continuously. ${ }^{5}$ The fall time of the neutron signal depends on the time of flight of the energetic ions reaching the target after the power supply is turned off. For example, if the threshold energy for deuterium ions to generate neutrons is $30 \mathrm{keV}$, and the acceleration gap is around $10 \mathrm{~cm}$, the transit time is approximately $40 \mathrm{~ns}$. Although it is possible to achieve a pulse fall time less than $1 \mu \mathrm{s}$, it requires an expensive and bulky high voltage switch thus making it not an attractive option.

\section{Beam Pulsing By Beam Chopping}

Fast pulsing can also be achieved by sweeping a beam across an aperture or a knife-edge, which is called "chopping"6,7 This method was used to generate a short pulse (pulse width $\sim 5 \mathrm{~ns}$ ) for pulsed fast neutron transmission spectroscopy application. ${ }^{8}$ To keep the neutron background low, two-stage acceleration is required. The beam is first accelerated to a medium energy for transverse sweeping. After going through the collimator, it is then further accelerated to full energy for neutron generator. The energy of the beam going through the collimator has to be kept low enough to minimize the background neutron noise. In this scheme, the switching time would be determined by the speed of transverse sweeping and the beam size with respect to the aperture of the knife-edge. The requirement of using extra electrodes and fast pulsing high voltage power supply to sweep the beam makes the system complicate and limits the overall current density and duty factor.

\section{Beam Pulsing By Controlling Extraction}

Figure 1 shows the most common scheme for beam switching by controlling the extraction gap. Forward bias will extract ions; reverse bias will stop ion flow but extract electrons. The switch-off times is mainly limited by the relatively slow velocity of the ions in the extraction gap and the plasma/beam boundary behavior at the source aperture. At the beam "off" state, the positive bias voltage applied to the extraction electrode extracts electrons from the ion source plasma and may bombard on the extraction electrode causing damages due to poor optics. The electrons can also ionize the background gas in the channel and create a local plasma. Ions leaking downstream will cause background neutrons.

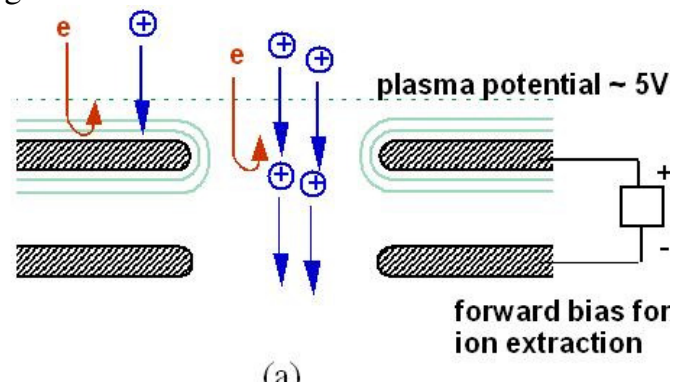

(a)

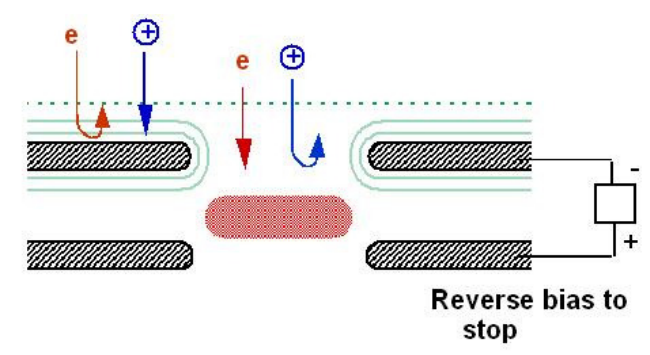

(b)

FIGURE 1. Schematic of beam pulsing by controlling the extraction. (a) Forward bias will extract ions; (b) reverse ions will stop ion flow but also pull electrons out from the ion source.

It is believed that if the aperture size is small enough (in the order of micrometer and much less than the plasma sheath thickness), most electrons can't escape even at reverse bias. However for micro-size aperture, the thickness of the extraction electrode will be on the order of ten microns (in order keep the proper aspect ratio), which makes it fragile and less heat resistant.

\section{Beam Pulsing By Retarding Potential (Gating)}

Gating the beam by a retarding potential is similar to the situation of controlling extraction. The difference is that the electrons are already separated out in the first gap (Figure 2). Forward bias in the second gap will allow the extracted ions to continue acceleration. Reverse bias will stop the ion flow. The fall time depends on the ion energy and retarding field strength. Figure 3 is a schematic drawing of the electrode structure. To the first order approximation, 
the fall time of the beam $\Delta \mathrm{t}$ is the time of flight of the ions with energy of $q V_{l}$ travelling across the gap distance L:

$$
\Delta t=\Delta v / a
$$

where $q$ is the charge, $\Delta v$ is the difference of the ion velocity before entering and after exiting the gap $\mathrm{L}, a$ is the acceleration of the ions under the field strength in the retarding field. Here $\Delta v$ can be calculated using the Equ. (2),

$$
\Delta v=\left(2 m V_{l} / q\right)^{1 / 2}
$$

where $m$ is the ion mass, $V_{l}$ is the beam extraction voltage; and $a$ can be calculated using Equ. (3),

$$
a=q E / m
$$

where $\mathrm{E}$ is the field strength in the retarding field. The fall time of the beam pulse is

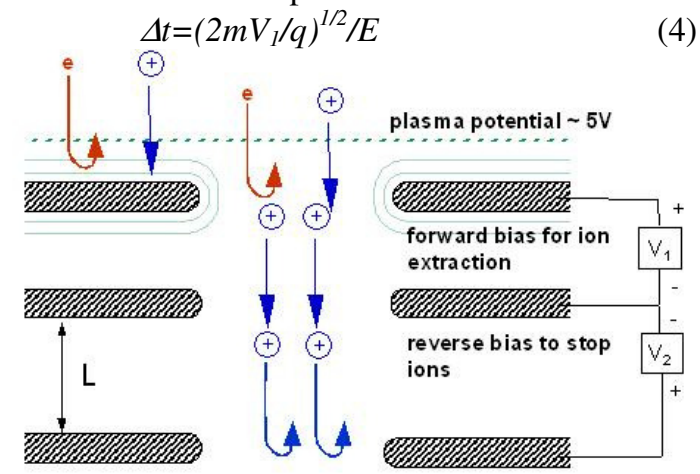

FIGURE 2. Schematic of beam pulsing by gating the ions at the second gap after separating out the electrons.

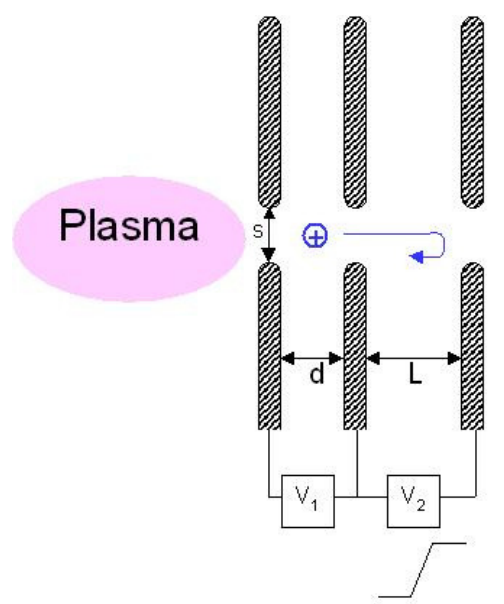

FIGURE 3. Schematic of the extraction system for gating the ion beam. In the drawing, $s$ is the diameter of the extraction aperture, the distance of extraction gap is d, and the length of retarding field is $\mathrm{L} . \mathrm{V}_{1}$ is the extraction voltage and $\mathrm{V}_{2}$ is the gating voltage.

It is interesting to note that the fall time of the beam pulse $\Delta \mathrm{t}$ is proportional to the square root of $V_{l}$ and inversely proportional to the retarding field $\mathrm{E}$. Figure 4 shows the beam fall time $\Delta \mathrm{t}$ at different extraction voltage $V_{l}$ from $100 \mathrm{~V}$ to $1 \mathrm{kV}$, as a function of retarding field strength E. Provided that the rise time of the gate pulser is negligible, a beam pulse with fall time less than $10 \mathrm{~ns}$ is possible, under reasonable retarding field strength $(<50 \mathrm{kV} / \mathrm{cm})$.

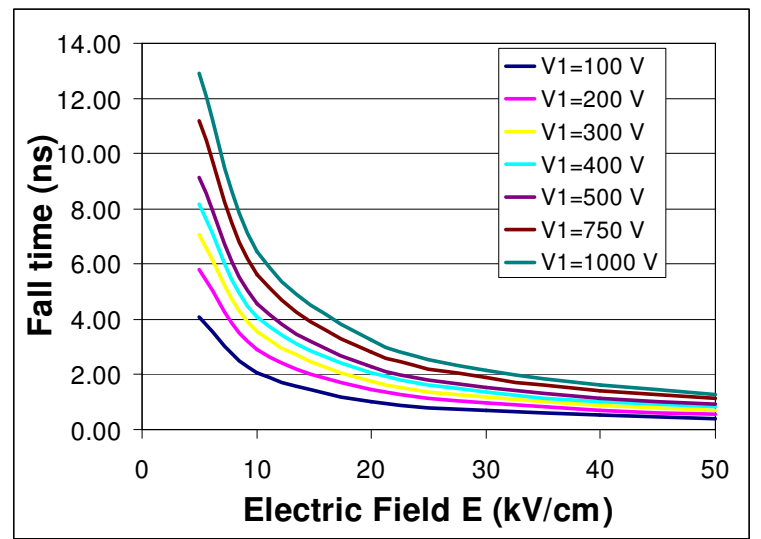

FIGURE 4. Calculated fall-off time of beam pulse as a function of retarding electric field $\mathrm{E}$. The beam extraction energy varies from $100 \mathrm{eV}$ to $1000 \mathrm{eV}$.

Retarding field strength $\mathrm{E}$ is determined by the gating voltage $V_{2}$ and the gap $\mathrm{L}$, as shown in the following equation.

$$
E=V_{2} / L
$$

Gating voltage $V_{2}$ doesn't need to be much higher than the extraction voltage $V_{l}$ to stop the ion flow. Therefore, the accelerator column can be miniaturized to extract the ions at low voltage. Using low voltage also minimizes the problem of voltage breakdown as well as reducing the time of flight to produce fast gating. In addition, low voltage is preferred because it not only makes the pulsing instrumentation easier, but also results in lower stored energy.

Table 1. Lists of corresponding ion current density for

\begin{tabular}{|c|c|c|c|c|c|c|c|}
\hline \multirow{2}{*}{\multicolumn{2}{|c|}{$\begin{array}{l}\text { Current density } \\
J\left(\mathrm{~mA} / \mathrm{cm}^{2}\right)\end{array}$}} & \multicolumn{6}{|c|}{ Extraction gap $d(\mathrm{~mm})$} \\
\hline & & 0.2 & 0.4 & 0.6 & 0.8 & 1 & 1.2 \\
\hline \multirow{7}{*}{$V_{1}(\mathrm{~V})$} & 100 & 96.30 & 24.07 & 10.70 & 6.02 & 3.85 & 2.67 \\
\hline & 200 & 272.37 & 68.09 & 30.26 & 17.02 & 10.89 & 7.57 \\
\hline & 300 & 500.37 & 125.09 & 55.60 & 31.27 & 20.01 & 13.90 \\
\hline & 400 & 770.37 & 192.59 & 85.60 & 48.15 & 30.81 & 21.40 \\
\hline & 500 & 1076.62 & 269.16 & 119.62 & 67.29 & 43.06 & 29.91 \\
\hline & 750 & 1977.88 & 494.47 & 219.76 & 123.62 & 79.12 & 54.94 \\
\hline & 1000 & 3045.15 & 761.29 & 338.35 & 190.32 & 121.81 & 84.59 \\
\hline
\end{tabular}
various extraction voltages $V_{1}$ and gaps $d$. The highlighted number represents the typical operating current density for available ion source.

Extraction voltage $V_{l}$ affects the current density of the ion source. According to the Child-Langmuir equation, current density,

$$
J=(4 / 9) \varepsilon_{0}(2 q / m)^{1 / 2} V_{l}^{3 / 2} / d^{2}
$$

where $\varepsilon_{0}$ is the permittivity of free-space, $V_{l}$ is the extraction voltage, and $\mathrm{d}$ is the distance of extraction gap. Table 1 lists the corresponding current density at different extraction $V_{l}$ from $100 \mathrm{~V}$ to $1 \mathrm{kV}$ and extraction gap from $0.2 \mathrm{~mm}$ to $1.2 \mathrm{~mm}$. For example, 
for an extraction voltage of $400 \mathrm{~V}$, one can use a gap of $0.8 \mathrm{~mm}$ to extract beam from an ion source of approximately $50 \mathrm{~mA} / \mathrm{cm}^{2}$. For normal beam optics, the diameter of an extraction aperture is slightly smaller than gap d. Therefore, we concluded that the best approach for a compact neutron generator to achieve less than $1 \mu \mathrm{s}$ pulse fall time is by gating multiple small ion beamlets at low energy. The total beam current requirement can be met by using multiple beamlets.

\section{EXPERIMENTAL SETUP}

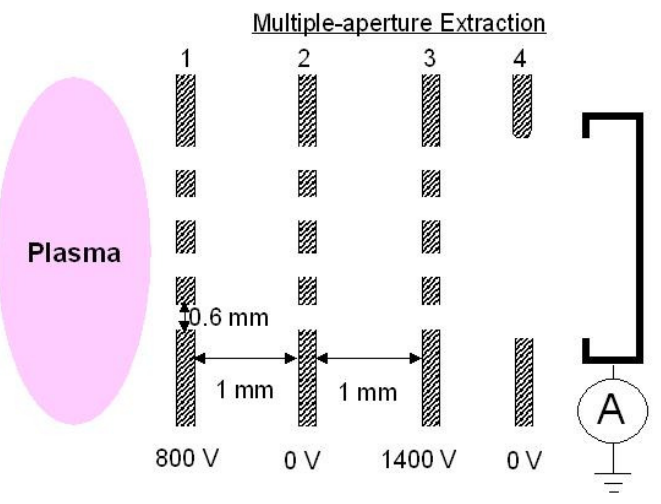

(a)

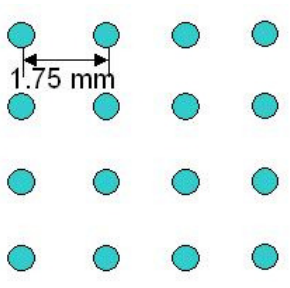

(b)

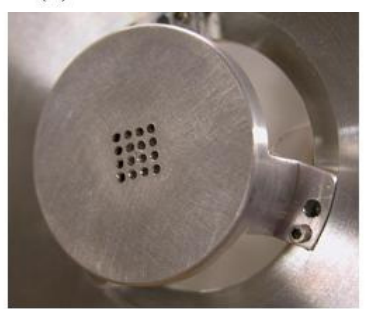

(c)
FIGURE 5. (a) Schematic drawing of the experimental setup for the beam pulse measurement. A multiple-aperture system is used to extract the ions from the plasma. (b) Front view of the array of $4 \times 4$ apertures. The diameter of each aperture is $0.6 \mathrm{~mm}$, and the center-to-center distance of each adjacent aperture is $1.75 \mathrm{~mm}$. (c) A photograph of the extraction system.

A multiple-aperture extraction system has been used in the beam pulse characteristics measurement. Figure 5 illustrates the experimental setup. A hydrogen plasma was generated using a $2.45 \mathrm{GHz}$ microwavedriven ion source. The detailed description of the ion source was reported by J. Kwan et al in Ref. 9. The plasma chamber is $9.2 \mathrm{~cm}$ in diameter and $12.7 \mathrm{~cm}$ long. Microwave power is transmitted via a rectangular waveguide through an aluminum nitride window into the plasma chamber. An axial magnetic field, that is required to set up the electron cyclotron resonant condition, is produced by passing approximately $109 \mathrm{~A}$ of dc current through the field coils. In the experiment, the microwave power was at $500 \mathrm{~W}$ and the gas flow was at $0.5 \mathrm{sccm}$. Hydrogen ions are extracted from an array of $4 \times 4$ apertures with each aperture $0.6 \mathrm{~mm}$ in diameter. The center-to-center distance between adjacent apertures is $1.75 \mathrm{~mm}$ thus the grid transparency is around $13 \%$. The extraction voltage $\mathrm{V}_{1}$ was varied from $400 \mathrm{~V}$ to $1000 \mathrm{~V}$, and the peak voltage of gating power supply $\mathrm{V}_{2}$ was set to 1400 V. The rise-time of gating pulse was approximately $500 \mathrm{~ns}$, and the pulse width was $10 \mu \mathrm{s}$. The fourth electrode, at ground potential, was inserted to reduce the capacitive couple current in the Faraday cup induced by the gating voltage. All the beams are collected by a Faraday cup, as shown in Figure 5(a). In order to minimize the effect of ground loop, the signal from the Faraday cup and its ground shielding case were fed into a unit-gain differential amplifier through a twist-pair co-axial cable.

\section{RESULTS AND DISCUSSIONS}

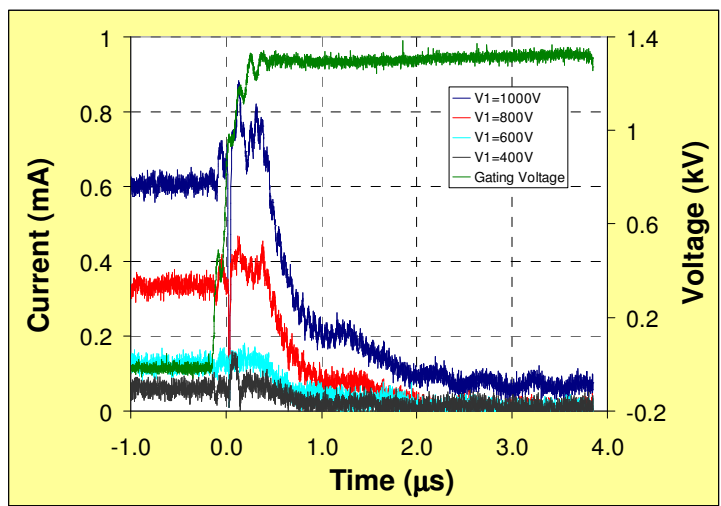

FIGURE 6. Measured pulsed beam current as a function time. The extraction voltage varies from $400 \mathrm{~V}$ to $1000 \mathrm{~V}$, and the gate voltage was set at approximately $1400 \mathrm{~V}$.

Results of the hydrogen ion beam current as a function of time are shown in Figure 6, with extraction beam energy varying from $400 \mathrm{eV}$ to $1000 \mathrm{eV}$. The background noise introduced by the capacitive-couple current (with no plasma running) has been subtracted from the signal. For each beam pulse, the current doesn't start to drop until the gate voltage stabilizes at approximately $1400 \mathrm{~V}$. There is a current increase immediately after the gate voltage is switched on, which is probably caused by beam bunching effect. As the gate voltage rises from zero to $1400 \mathrm{~V}$, an electric field built up between Electrodes 2 and 3, which is the retarding field for stopping the ion flow. Between Electrodes 3 and 4, an electric field is built up as well. In this region, those ions that have exit the gate electrode (\#3) will be accelerated and results in a signal increase in the Faraday cup for a short period. 
Figure 7 plots the measured fall time (dropping to $20 \%$ of the full current level) of extracted ion beam pulses at beam energies between $400 \mathrm{eV}$ to $1000 \mathrm{eV}$, with the gate voltage set at approximately $1400 \mathrm{~V}$. Except for the case of extraction voltage at $1000 \mathrm{~V}$, the beam fall time for low voltage extraction (from $400 \mathrm{~V}$ to $800 \mathrm{~V}$ ) are less than $1 \mu \mathrm{s}$. Nevertheless, these experimental results are significantly larger than the values calculated in Section II. To explore the actual limit of the pulse fall-off time, there are several aspects of the experimental setup that can be improved. First of all, the Faraday cup used in the experiment was not configured to transmit fast pulse signal. Secondly, the co-axial cable in the setup can be further shortened to reduce $R C$ delay in the circuitry. And finally, the noise of capacitive-coupled signal in the Faraday cup needs to be minimized.

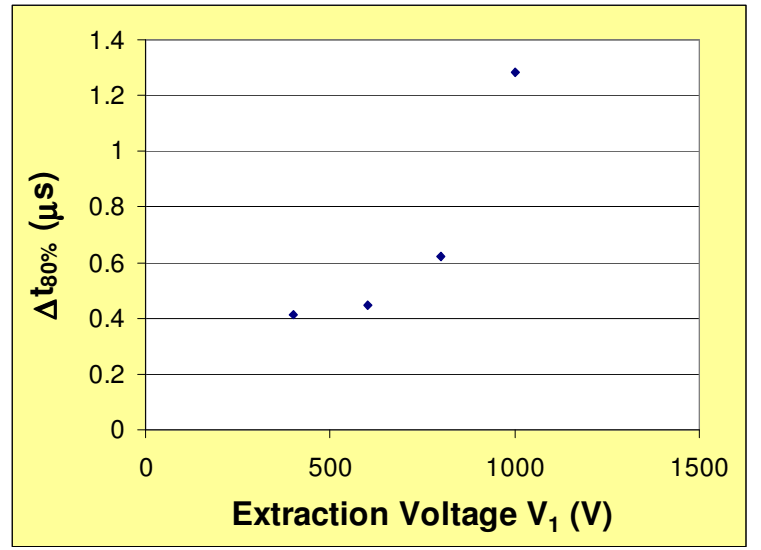

FIGURE 7. The fall-off time (dropping to $20 \%$ of the full current level) for the beam pulse as a function of extracted beam voltage.

The goal of this development is to build a fast falltime pulsed neutron generator with the following features: pulse width of tens of $\mu \mathrm{s}$, fall time less than 1 $\mu \mathrm{s}$, repetition rate up to $10 \mathrm{kHz}$, and the peak DD neutron yield as high as $10^{9} \mathrm{n} / \mathrm{s}$. To achieve $10^{9} \mathrm{n} / \mathrm{s}$ D$\mathrm{D}$ neutrons, $10 \mathrm{~mA}$ of $\mathrm{D}^{+}$ions are required. If the current density of the deuterium ion source is 50 $\mathrm{mA} / \mathrm{cm}^{2}$, the extraction area needs to be $0.2 \mathrm{~cm}^{2}$. It takes 400 apertures to obtain this total area, if the diameter of each aperture is $0.25 \mathrm{~mm}$. The center-tocenter distance of the adjacent apertures can be as big as three times of the aperture size, i.e. $0.75 \mathrm{~mm}$, considering that the aperture size for the downstream electrodes may be larger than the plasma electrode and engineering limitation in terms of how close the apertures can be machined. The extraction region covers a region of $14.5 \mathrm{~mm} \times 14.5 \mathrm{~mm}$, with a transparency of approximately $12 \%$. Usually merging an array of beamlets suffers the loss of beam brightness, i.e., emittance growth, but that is not an important issue for neutron source applications. The effective current density of the source dropping from $50 \mathrm{~mA} / \mathrm{cm}^{2}$ to $6 \mathrm{~mA} / \mathrm{cm}^{2}$ can actually reduce the heat power density on the target, making the target engineering easier.

\section{SUMMARY}

Different methods of achieving fast fall time ion beam pulses have been evaluated, we concluded that the best approach to achieve pulse fall time less than 1 $\mu \mathrm{s}$ is by using retarding potential to gate multiple ion beamlets at low energy. Beam fall time less than $1 \mu \mathrm{s}$ using an array of $4 \times 4$ beamlets has been demonstrated. We'll continue the beam pulse shape measurement and explore the limit of pulse fall-time. The design of prototype fast fall-time pulsed neutron generator using an array of $20 \times 20$ beamlets is in progress. The detail design and fabrication and results of testing the prototype neutron generator will be reported in future publication.

\section{ACKNOWLEDGMENTS}

The authors would like to thank Dr. Bernhard Ludewigt for his helpful discussions, and Will Waldron, Tom McVeigh, and Paul Wong for their technical support. This work is supported by NA22 of NNSA under the Department of Energy contract No. DE-AC02-05CH11231.

\section{REFERENCES}

1. B.D. Rooney et al., IEEE Nuclear Science Symposium, Conference Record, 2, 1027(1998).

2. K. A. Jordan, and T. Gozani, Nucl. Instr. and Meth. A 579, 388 (2007).

3. J. D. Grow and J. S. Foster Jr., Rev. Sci. Instr., 24, 606 (1953).

4. C. E. Anderson and K. W. Ehlers, Rev. Sci. Instr., 27, 809 (1956).

5. K. H. Beckurts, Nucl. Instr. Methods, 11, 144 (1961).

6. T. K. Fowler and W. M. Good, Nucl. Instr. Methods, 7, 245 (1960).

7. C. D. Moak, W. M. Good, R. F. King, J. W. Johnson, H. E. Banta, J. Judish, W. H. duPreez, Rev. Sci. Instr., 35, 672 (1964).

8. T. Kalvas, S. K. Hahto, F. Gicquel, M. King, J. H. Vainionpaa, J. Reijonen, and K. N. Leung, Rev. Sci. Instr., 77, 03B904 (2006).

9. J. W. Kwan, R. Gough, R. Keller, B. A. Ludewigt, M. Regis, R. P. Wells, and J. H. Vainionpaa, "A $2.45 \mathrm{GHz}$ High Current Ion Source for Neutron Production", 17th International Workshop on ECR Ion Sources and Their Applications, September 17-21, 2006, IMP, Lanzhou, China. 